\title{
Difficulty Diagnosing a Brain Tumor during Clinical Maintenance of a Complete Response to anti-HER2 Treatments for Metastatic Breast Cancer: A Case Report
}

\author{
Ryoko Semba $^{a}$ Yoshiya Horimoto ${ }^{a, b}$ Atsushi Arakawa ${ }^{b}$ Yoko Edahiro ${ }^{c}$ \\ Tomoiku Takakuc Kotaro lijima ${ }^{\mathrm{a}}$ Mitsue Saito $^{\mathrm{a}}$ \\ aDepartment of Breast Oncology, Juntendo University School of Medicine, Tokyo, Japan; \\ bDepartment of Human Pathology, Juntendo University School of Medicine, Tokyo, Japan; \\ 'Department of Hematology, Juntendo University School of Medicine, Tokyo, Japan
}

\section{Keywords}

Advanced breast cancer - Brain tumor - Diffuse large B-cell lymphoma of central nervous system · Pathology

\begin{abstract}
A 46-year-old woman with erythema of the right breast presented to our hospital and was diagnosed with stage IV breast cancer (HER2-positive invasive ductal carcinoma). She received 4 courses of anthracycline-based regimens and 4 courses of trastuzumab + pertuzumab + docetaxel (Tmab + Pmab + DTX).

Since she responded well to these therapies, only Tmab + Pmab was continued thereafter. Twenty-three months after starting treatment, she developed a headache. A tumor was identified in the right temporal lobe. Craniotomy was performed for definitive diagnosis. Intraoperative pathological assessment suggested the tumor to be brain metastasis of breast cancer. However, the final pathological diagnosis was diffuse large B-cell lymphoma of central nervous system (DLBCL-CNS) based on re-assessment with immunohistochemical examinations. Therefore, the Tmab + Pmab was discontinued, and 6 courses of high-dose methotrexate therapy were administered. This case highlights the importance of considering rare entities, such as DLBCL, when diagnosing a solitary brain tumor in a patient with a primary cancer, based on imaging and pathological findings.




\section{Introduction}

In the treatment of HER2-positive metastatic breast cancer (MBC), combinations of chemotherapeutic agents and anti-HER2 drugs are administered. Trastuzumab (Tmab) has long played a major role in these treatment regimens, but survival rates have recently been further extended by induction strategies including pertuzumab (Pmab), Trastuzumab emtansine (T-DM1) [1, 2]. With these treatment regimens, some HER2-positive MBC patients can obtain good tumor control and maintain a clinically partial response or even a complete response (CR) over the long term.

Patients with HER2-positive breast cancer often develop brain metastasis. Occurrence rates of brain metastasis reportedly range from 30 to $44 \%$ [3]. Suggested reasons for the high occurrence of brain metastasis include HER2-positive breast cancer possibly having an inherent biological tendency to metastasize to the brain, via an organ-specific molecular mechanism [4]. Another possibility is the presumably poor permeability of anti-HER2 therapeutic agents due to the blood-brain barrier $[5,6]$.

In most cases, more than one metastatic brain tumor develops, but if a brain tumor is solitary, it can be difficult to determine whether it is primary or metastatic [7]. On imaging, a ring-shaped lesion is often observed on computed tomography (CT) or magnetic resonance imaging (MRI) scans. Such lesions require a meticulous differential diagnosis considering glioblastoma, malignant lymphoma of the central nervous system, and brain abscess. When it is difficult to make a diagnosis based only on imaging findings, a craniotomy is often performed to obtain a definitive diagnosis.

We herein describe a patient in whom diagnosing a primary malignant lymphoma, which developed during maintenance of a clinical CR to anti-HER2 treatments for MBC, was challenging.

\section{Case Presentation}

A 46-year-old Japanese woman came to our hospital with a chief complaint of redness of the right breast. Her family history was unremarkable. Her past history included epilepsy and she thus regularly visited the Neurology Department of our Hospital. Physical examination at

Fig. 1. Pathological findings of right breast core needle biopsy. Atypical cells with distorted nuclei proliferate in the form of cords with the growth of interstitial components, while cells forming a glandular cavity are few in number.

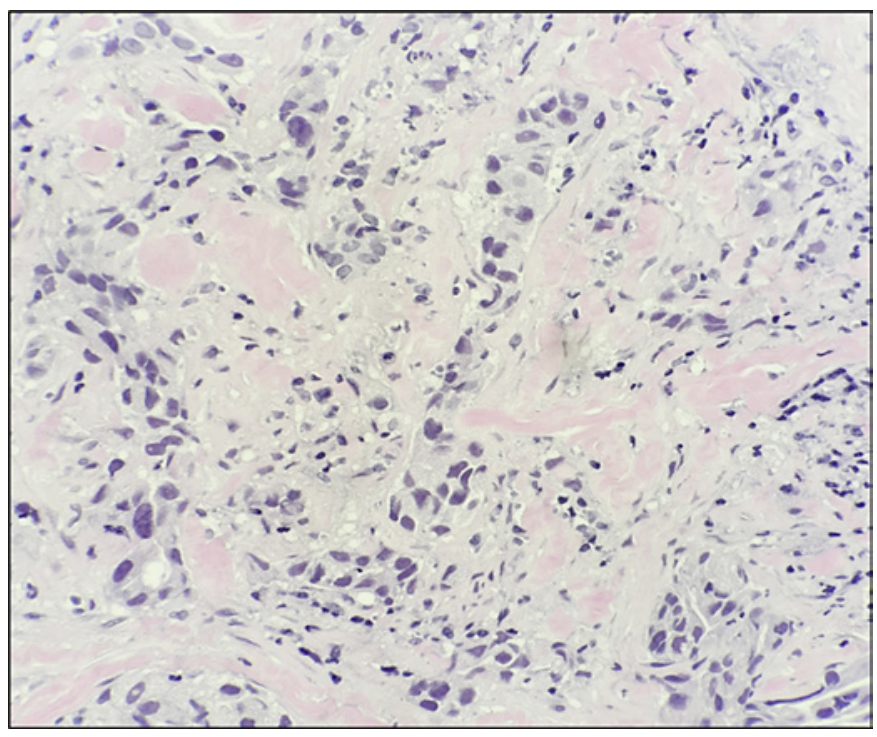


Fig. 2. Changes in PET-CT imaging during anti-HER2 treatments. a At the start of treatment. b 23 months after starting of treatment. Hot spots of the primary lesion of the right breast, axilla, anterior mediastinum, and left pleura disappeared in response to treatment.
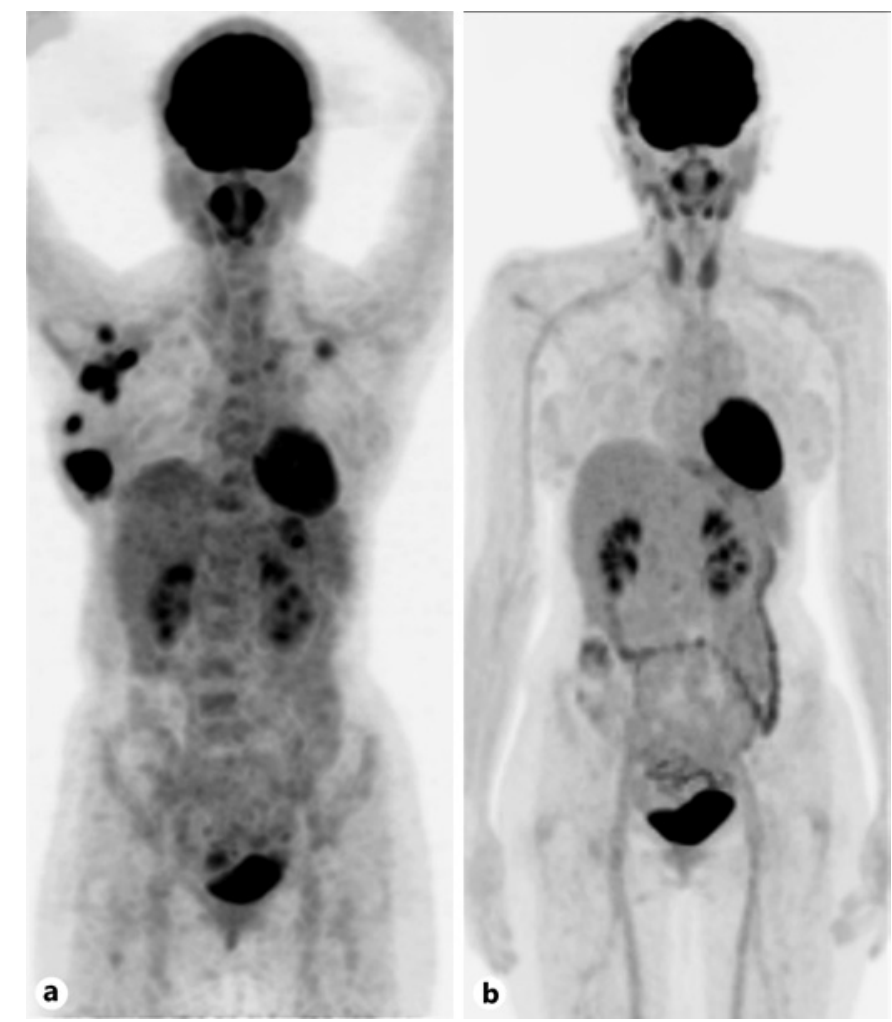
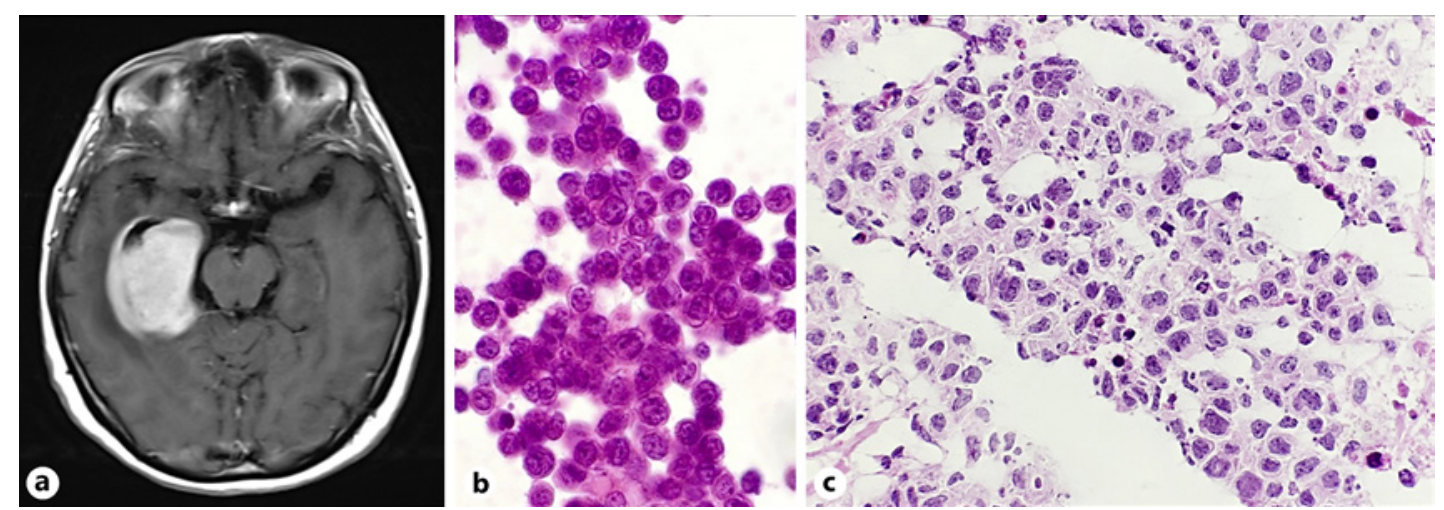

Fig. 3. Brain MRI and intraoperative histological assessment. Brain MRI examination (T1-weighted; a) and intraoperative pathological findings (stamp specimen, b; frozen section, c) are shown. Atypical cells proliferate densely in the imprint cytology, and intercellular connectivity and lumen-like structures are also seen. In frozen specimens, gap formation and cells with strong atypical growth proliferating in alveolar nests are observed.

the first visit revealed erythema and swelling mainly in the lower part of the right breast. Ultrasound revealed a low-echo $54 \times 41 \times 31 \mathrm{~mm}$ mass in the outer area, with a partially unclear boundary. Ultrasound-guided needle biopsy was performed, and histological examination revealed invasive ductal carcinoma (no special type), high grade, $\operatorname{ER}(+), \operatorname{PgR}(-)$, HER2(3+), Ki67 L.I. 70\% (Fig. 1). Whole-body positron emission tomography (PET)-CT scanning was performed to detect possible distant metastases and showed an increased uptake in the right axilla, anterior mediastinum, left pleura, and liver (Fig. 2a). Based on these 


\section{Case Reports in Oncology}

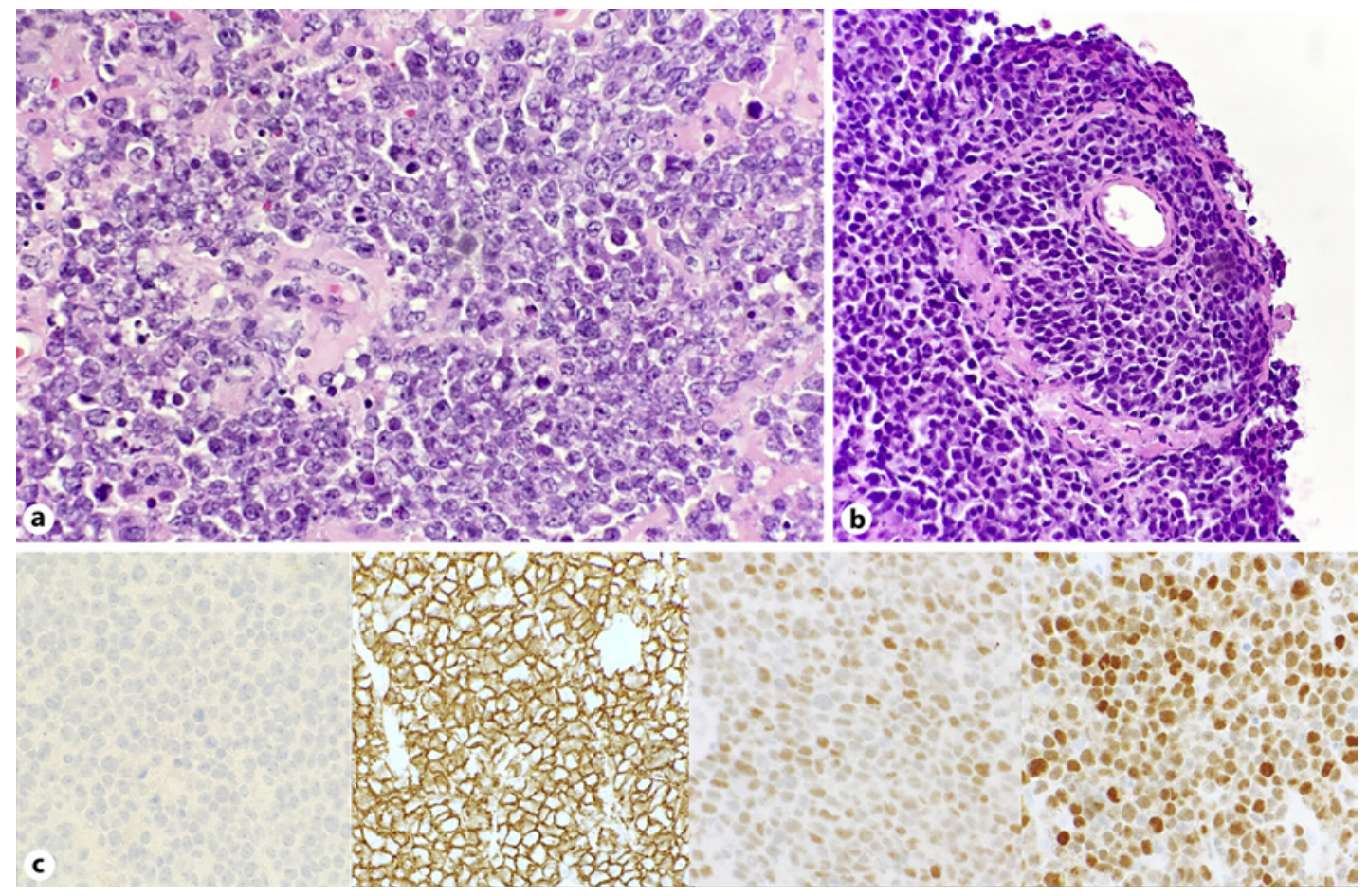

Fig. 4. Histological assessment of the permanent specimen. a Dense proliferation of atypical cells with large nuclei, but lacking intercellular connectivity. b Proliferation around vessel, typical findings of DLBCL. c Immunohistochemical findings of CD10, CD20, bcl6, and MUM1 (from left to right).

findings, the diagnosis was cT4dN3M1 stage IV breast cancer. After 4 courses of FEC (fluorouracil, epirubicin, cyclophosphamide) therapy, the lesions had nearly disappeared. After 4 courses of Tmab + Pmab + DTX therapy, clinical CR was maintained for 23 months after starting Tmab + Pmab therapy (Fig. 2b).

Two years after starting treatment, she complained of a persistent headache. CT and MRI scans of the head revealed a 30-mm mass lesion in the right temporal lobe (Fig. 3a). Consulting with our neurosurgery team, brain metastasis of the breast cancer was considered the mostly likely diagnosis, based on the clinical course and imaging findings. A craniotomy for tumor resection was performed to obtain a definitive diagnosis. Intraoperative pathological assessment of frozen sections suggested brain metastasis of breast cancer (Fig. 3b, c). Since the tumor was partially exposed in the cerebrospinal fluid cavity when the operation was performed, wholebrain irradiation was started 2 weeks after the operation. However, the final pathological diagnosis was diffuse large B-cell lymphoma of the central nervous system (DLBCL-CNS), obtained with additional immunohistochemistry (CD3(-), CD5(+), CD10(-), CD20(+), bcl2(+), bcl6(+), MUM1(+), Ki67 L.I. 90\%) (Fig. 4). Thus, radiation therapy was promptly discontinued and high dose-methotrexate( 6 courses) for DLBCL-CNS was administered.

\section{Conclusion}

DLBCL-CNS is a subtype of lymphoma that forms lesions confined solely to the central nervous system. DLBCL-CNS shows very rapid progression and has a poor prognosis [8]. Regarding MRI findings, the tumor typically shows an equal to low signal on T1-weighted 
images, a mild to high signal on T2-weighted images [9], and a strong uniform contrast effect when a contrast agent is administered. Marked cerebral edema is observed around the lesion. It may be difficult to differentiate DLBCL-CNS from glioblastoma or metastatic brain tumor. Pathological assessment with surgical biopsy is thus necessary.

In our case, the intraoperative pathological findings on frozen sections were similar to those of MBC, making difficult to obtain a final diagnosis of DLBCL. The patient's clinical course also suggested MBC. Surgeons should be aware that pathological diagnosis, especially intraoperative assessment, is often challenging. Such cases require close collaboration between radiologists and pathologists.

\section{Acknowledgements}

The authors sincerely appreciate Dr. Bierta Barfod for her help with the language editing.

\section{Statement of Ethics}

Written informed consent was obtained from the patient and her parents for publication of this case report.

\section{Conflict of Interest Statement}

The authors declare that they have no competing interests in any aspect of this case.

\section{Funding Sources}

The authors declare that they have received no financial support pertaining to this report.

\section{Author Contributions}

R.S., K.I., and M.S. treated the patient for metastatic breast cancer.Y.H. and A.A. conducted the histological assessments. Y.E. and T.T. treated the patient and provided clinical information. R.S. and Y.H. wrote the manuscript. M.S. reviewed and edited the manuscript. All authors contributed to discussions and agreed on the final version of the manuscript for submission.

\section{References}

1 Baselga J, Cortés J, Kim SB, Im SA, Hegg R, Im YH, et al. Pertuzumab plus Trastuzumab plus Docetaxel for Metastatic Breast Cancer. N Engl J Med. 2011;366(2):109-19.

2 Diéras V, Miles D, Verma S, Pegram M, Welslau M, Baselga J, et al. Trastuzumab emtansine versus capecitabine plus lapatinib in patients with previously treated HER2-positive advanced breast cancer (EMILIA): a descriptive analysis of final overall survival results from a randomised, open-label, phase 3 trial. Lancet Oncol. 2017;18(6):732-42.

3 Tonyali O, Coskun U, Yuksel S, Inanc M, Bal O, Akman T, et al. Risk factors for brain metastasis as a first site of disease recurrence in patients with HER2 positive early stage breast cancer treated with adjuvant trastuzumab. Breast. 2016;25:22-6. 
4 Pestalozzi BC, Zahrieh D, Price KN, Holmberg SB, Lindtner J, Collins J, et al. Identifying breast cancer patients at risk for Central Nervous System (CNS) metastases in trials of the International Breast Cancer Study Group (IBCSG). Ann Oncol. 2006;17(6):935-44.

5 Brufsky AM, Mayer M, Rugo HS, Kaufman PA, Tan-Chiu E, Tripathy D, et al. Central nervous system metastases in patients with HER2-positive metastatic breast cancer: incidence, treatment, and survival in patients from registHER. Clin Cancer Res. 2011;17(14):4834-43.

6 Gabos Z, Sinha R, Hanson J, Chauhan N, Hugh J, Mackey JR, et al. Prognostic significance of human epidermal growth factor receptor positivity for the development of brain metastasis after newly diagnosed breast cancer. J Clin Oncol. 2006;24(36):5658-63.

7 Patchell RA, Tibbs PA, Walsh JW, Dempsey RJ, Maruyama Y, Kryscio RJ, et al. A randomized trial of surgery in the treatment of single metastases to the brain. N Engl J Med. 1990;322(8):494-500.

8 Perini GF, Campregher PV, Santos FP, Hamerschlak N. Primary central nervous system lymphoma: what a neurologist/neurosurgeon should know? Arq Neuropsiquiatr. 2013;71(4):254-7.

9 Meyer H-J, Schob S, Münch B, Frydrychowicz C, Garnov N, Quäschling U, etal. Histogram analysis of T1-weighted, T2-weighted, and postcontrast T1-weighted images in primary CNS lymphoma: correlations with histopathological findings—a preliminary study. Mol Imaging Biol. 2018;20(2):318-23. 